\title{
Improving the Quantum Efficiency of Photocathode CsI
}

\author{
Zhang Xuanni \\ Institute of Physics and Electronic Engineering, \\ Xianyang normal University \\ Shanxi, Peoples Republic of China. \\ Moishel@163.com
}

\author{
Lu Fan \\ Yangling Metron New Material Co.,Ltd, \\ Xian yang, Peoples Republic of China \\ lufan@ylmetron.com
}

\begin{abstract}
CsI is a great photoelectric emission material in the field of photon imaging detectors owing to its high quantum efficiency in the ultraviolet range. But quantum efficiency depends on the electron emission mode deeply. With specific structure of photocathode, the photoelectron can operate in both semitransparent mode and reflective mode. So some experiments were done to verify which mode is better and how to get it. Theory and experiment show that the quantum efficiency in reflective mode is higher than Quantum Efficiency in semitransparent mode. For a certain specifications of the vacuum tube, the parameter of reflective mode fabrication was obtained. Particularly in double proximity focusing image intensifier tube system, CsI photocathode can be deposited on micro channel plate(MCP), and avoid applying high voltage between the incident window and MCP, which is micro distance. Above all, this enable photoelectric emission to operate in the reflective mode, thus higher quantum efficiency can be obtained. The thickness of CsI film influence the quantum efficiency and it depend on the assembly structure of MCP. Some theory analysis and experiment proved it in this paper.
\end{abstract}

Keywords-Ultraviolet; CsI photocathode; Quantum Efficiency; reflective mode; $\mathrm{MCP}$

\section{INTRODUCTION}

Ultraviolet detection technology rapidly development in recent years, but the sensitivity and detection distance are the main bottleneck influence Ultraviolet detection technology development ${ }^{[1]}$. While the two indexes closely depend on optoelectronic cathode quantum efficiency, how to improve the efficiency of the cathode ultraviolet light quantum is the key of resolving the problem. CsI is a material of great interest in the field of photon imaging detectors owing to its high quantum efficiency in the ultraviolet range $1.15 \sim 2 \times 10^{-7} \mathrm{~m}$, but the thickness of CsI photocathode and the mode of photoemission influence the quantum efficiency. According semitransparent CsI photoemission theory, that films thinner than the electron escape length are characterized by a higher quantum efficiency with respect to thin films operating only in semitransparent mode(STM), or thick films operating in reflective mode(RFM) $)^{[2,3]}$. Since in STM the thickness of CsI photocathode is too thin to make the deposition of CsI layers uniform, the high quantum efficiency is hard to ensure. While the thickness of CsI photocathode is thicker in RFM than that

This work was financially supported by the National Natural Science Foundation of China (Grant No.61307002, 61275184), the Scientific Research Program(No.2XSYK017, No.12XSYK014), provincial college students' innovative entrepreneurial training program of xianyang normal university (2456). in STM, so it is more attractive ${ }^{[4]}$. In this paper semitransparent mode CsI films on optical input window substrate and reflective mode CsI films on substrates micro channel plate (MCP) is discussed in detail.

\section{STM ON OPTICAL INPUT WINDOW SUBSTRATES}

Ultraviolet signal general is weak, so photoelectrons emission of Ultraviolet photocathode does. In the Imaging Reverse Focus system, photoelectrons transit more time, it will diffuse more, image will be serious distortion ${ }^{[4,5]}$. Shorten the distance from the photocathode to the screen means reducing the photoelectrons transit time. Therefore, generally double proximity focusing image intensifier tube is more favourite, Fig1 shows the structure schematic diagram of double proximity focusing image intensifier tube, and it is a vacuum tube. In order to maximize the photoemission current to be measured, the photocathode is put as closely as possible to the fluorescent target. A multiplier consisting of two microehannel plates (MCP) behind the transmission photocathode amphfies the photoelectron signals to improve the measuring sensitivity. Optical input window $\mathrm{MgF}_{2}$ proximity MCP with $1 \sim 3 \times 10^{-4} \mathrm{~m}$, MCP proximity fluorescent screen with $5 \times 10^{-4} \mathrm{~m}$ or so. Then the pack frame ring and ceramic sealing up the component, the fabricating is hard to achieve this for so little size. The double proximity which has added more than $100 \mathrm{~V}$ voltages, that is easy to cause field-emission. Photocathode CsI deposited on the inner surface of optical input window MgF2 work in STM, about $6 \times 10^{-5} \mathrm{~m}$ very thin CsI layers get quantum efficiency, only about $25 \%-30 \%{ }^{[6]}$, as Fig2 shows. While in RFM, the quantum efficiency is higher than it in STM, and the thickness is not strictly limited. But photocathode CsI deposited on the inner surface of optical input window MgF2 can't implement photoemission by RFM, so some attempt to deposit photocathode CsI on substrates MCP directly, and the experiments is analyzed as the following. 


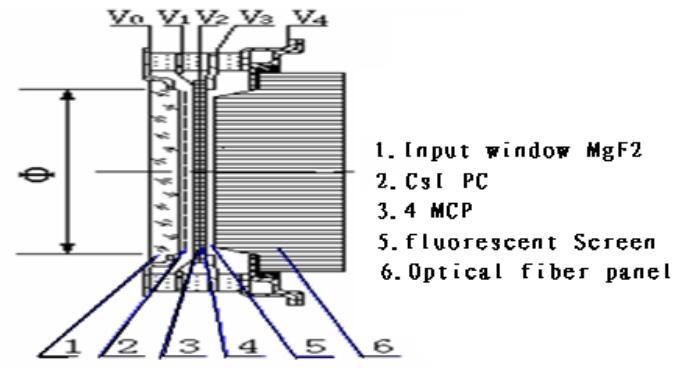

Fig. 1. Structure schematic diagram of double proximity focusing image intensifier tube

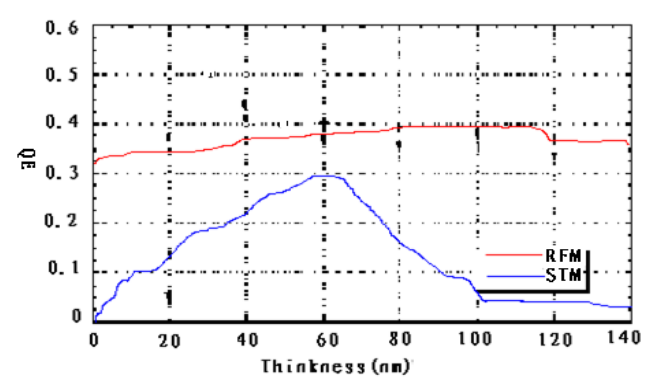

Fig. 2. The quantum efficiency variation with CsI film thickness

\section{RFM ON SUBSTRATES MCP}

CsI photocathode is deposited on substrates MCP directly, as Fig3 shows, it can operate both in STM and $\mathrm{RFM}^{[7,8]}$, which is determined by MCP type. The aperture of MCP channel is small with channel bending; it will easy operate in STM. But in order to achieve higher quantum efficiency, RFM is desired. So the type of MCP must be chosen carefully. The thickness of photocathode CsI layers influence quantum efficiency, above all, we optimized the thickness of the CsI photocathode. The quantum efficiencies were simultaneously monitored at a wavelength of $170 \mathrm{~nm}$ with vacuum-plated CsI. The optimized thickness was about $1300 \mathrm{~nm}$, and in RFM it is thicker than thickness of CsI layers in STM.

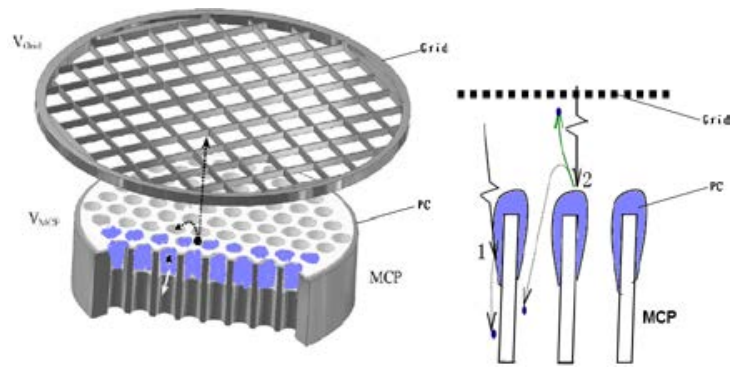

Fig. 3. The Schematic of photoelectron transmission on MCP substrates

If the aperture of MCP channel is small, CsI layers is thicker, the CsI layers will seal the channel tightly, photoelectrons will be difficulty to cross, then secondary electrons reduced, quantum efficiency will be lower. If the aperture of MCP channel is large, CsI layers is not thick enough, the CsI layers will operate STM, quantum efficiency of CsI photocathode will be lower too. So thickness of CsI layers should be chosen according the aperture of MCP channel size and shape, as Fig4 shows. The photoelectric effect and plating optoelectronic cathode is very randomness, so the value of thickness only base on plenty of experiments. We had done many experiments on MCP with different channel shape and size, got some experimental result showing in Fig5. Light source wavelength is $1.72 \times 10^{-7} \mathrm{~m}$, the aperture of MCP channel is $1.0 \times 10^{-5} \mathrm{~m}$, tilt angle is $12^{\circ}$, diameter of MCP is $\mathbf{3} \times \mathbf{1 0}^{-2} \mathrm{~m}$, MCP plate is divided into 4 areas. The thickness of CsI layers is $1.52 \times \mathbf{1 0}^{-6} \mathrm{~m}$ in $A, \quad \mathbf{0 . 9 1} \times \mathbf{1 0}^{-6} \mathrm{~m}$ in $B$, $\mathbf{1 . 3 8} \times \mathbf{1 0}^{-6} \mathrm{~m}$ in $\mathrm{C}, \quad \mathbf{2 . 1 0} \times \mathbf{1 0}^{-6} \mathrm{~m}$ in $\mathrm{D}$. The illumination of all area show in Fig5, the stronger illumination, the higher quantum efficiency.It easy to find that $\mathrm{C}$ area is optimal, that is the optimum thickness of the CsI photocathode is $\mathbf{1 . 3 8} \times \mathbf{1 0}^{-\mathbf{6}} \mathrm{m}$ in the condition of above-mentioned incident light wavelength and MCP type.
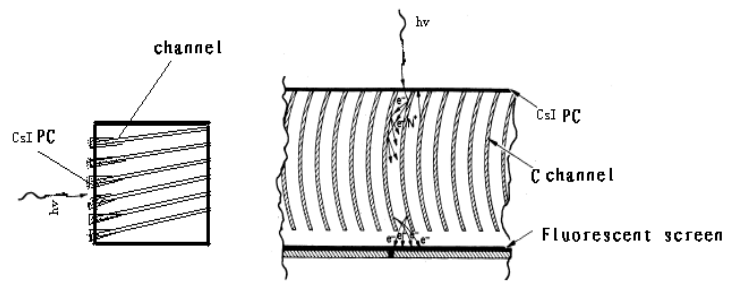

Fig. 4. The Schematic of photoelectron transmission in different channel shape of MCP
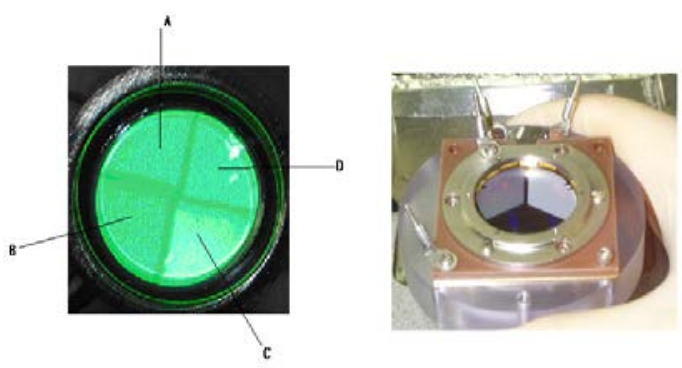

Fig. 5. The illumination of all areas A, B, C, D

\section{CONCLUSION}

(1)The reflective and semi-transparent CsI photoemission theory, we have shown that films thinner than the electron escape length are characterized by a higher quantum efficiency with respect to thin films operating only in semitransparent mode, or thick films operating in reflective mode.

(2)Photocathode CsI on optical input window substrates only operate in STM; but on MCP substrates can operate in both STM and RFM, in contrast, quantum efficiency of RFM is higher than STM.

(3)The thickness of CsI layers is adequate to one MCP model, it depend on the shape and size of MCP channel. When light source wavelength is $1.72 \times 10^{-7} \mathrm{~m}$, the aperture of MCP channel is $1.0 \times 10^{-5} \mathrm{~m}$, with tilt angle $12^{\circ}$ and diameter of 
MCP $\mathbf{3} \times \mathbf{1 0}^{-2} \mathrm{~m}$, the optimum thickness of the CsI photocathode is $\mathbf{1 . 3 8} \times \mathbf{1 0}^{-6} \mathrm{~m}$.

All the result of this paper paves the way for deeper research and engineering practice.

\section{ACKNOWLEDGEMENTS}

This study was supported the National Natural Science Foundation of China (No.61307002, 61275184). the Scientific Research Program (No.2XSYK017, No.12XSYK014), provincial college students' innovative entrepreneurial training program of xianyang normal university (2456). The authors would like to thank Dr. B.S. Zhao for the help with the manuscript preparation.

\section{REFERENCES}

[1] A.S.Tremsin, S.Ruvimov O.H.W. Siegmund, "Structural transformation of CsI thin film photocathodes under exposure to air and UV irradiation.” Nucl. Instr. and Meth.A vol. 447 2000.pp.614-618.
[2] H. Toru, T.Yoshihito, K Hideo, I.Tetsuya. "Performance of a CsI photocathode in a hard x-ray streak camera.” Rev. Scie. Instru., vol. 71.2000.pp.265-268.

[3] ZHANG XUAN-NI, ZHAO BAO-SHENG, ZHU XIANG-PING, PAN JUN-JIE. The Research of Photocathode CsI on MCP Substrate. Journal of Xianyang Normal University. vol. 6.2007.pp.69-73(In Chinese).

[4] Joseph C L Argabright, V. Bybee, R. Danks. A. and Woodgate.B: Proc. SPIE. Ultraviolet Technology V, 2282 (1994) 116.

[5] M.P. Kowalski, H. Gursky, J.C. Rife, D.J. Yentis, R.G. Cruddace, T.W. Barbee, Jr., W.H. Goldstein, J.F. Kordas, K.F. Heidemann, G.G. Fritz, W.R. Hunter, M.A. Barstow, G.W. Fraser, N.P. Bannister, J.S. Lapington, J.A. Tandy, and B. Sanderson: proc SPIE, 4498(2001)303.

[6] S. Schuh, S. Dreizler, and B. Wolff, Incident angle effect on the quantum efficiency of CsI photocathodes Spectrometers Detectors and Associated Equipment .vol.360(1)• June 1995 pp.430-431

[7] A.S.Tremsin, O.H.W. Siegmund, "Quantum efficiency and stability of alkali halide UV photocathodes in the presence of electric field” Nucl. Instrum. Meth. Vol.504, Issues 1-3, 21 May 2003, pp 4-8.

[8] FAN Long, LI Yu-Kun, CHEN Tao et al. "Recent Progress in Research on CsI Thin Film Photocathodes” .Journal of Inorganic Materials, 2015, 30(3): pp.225-232.

[9] A.S. Tremsin, O.H.W. Siegmund, IEEE Trans. Nucl. Sci. 2001, 48 (3):pp 421-428.

[10] A. Butler et al. Bio-medical X-ray imaging with spectroscopic pixel detectors, Nucl. Instrum. Meth. A, 2008, 591(1) : pp141-146. 